\section{Granulysin skin game}

\section{By Lauren Martz, Staff Writer}

Researchers in Taiwan have identified a cytotoxic protein, granulysin, that could be targeted to treat or diagnose Stevens-Johnson syndrome and toxic epidermal necrosis. ${ }^{1}$ The data suggest the protein is a central player in the skin cell necrosis seen in patients with these conditions.

Stevens-Johnson syndrome (SJS) and toxic epidermal necrosis (TEN) are cutaneous eruptions caused by adverse drug reactions and have a mortality rate of $10-50 \% .^{2}$ Mechanistically, both are considered variants in severity of the same condition: SJS is characterized by $<10 \%$ skin detachment, TEN by $>30 \%$ detachment and overlapping SJS and TEN by $10-30 \%$ detachment. ${ }^{3}$

The reactions have been linked to the use of many drugs, with most reports being related to anticonvulsants, antibiotics and NSAIDs. ${ }^{4}$ SJS risks contributed to the 2005 recall of Bextra valdecoxib, a cyclooxygenase-2 (RTGS2; COX2) inhibitor to treat arthritis from Pfizer Inc., and a negative FDA panel in 2006 for Sparlon modafinil from Cephalon Inc. to treat pediatric ADHD. ${ }^{5}$

Indeed, a therapeutic or diagnostic for SJS and TEN potentially could be used to help rescue drugs in the future.

Previous work has shown that the blister lesions resulting from SJS/ TEN contain infiltrating cytotoxic T lymphocytes (CTLs) and natural killer (NK) cells, and studies have suggested that granule-mediated release of cytotoxic proteins including perforin, granzyme B and Fas ligand (FasL) may contribute to keratinocyte cell death and necrosis. ${ }^{6}$

Standard care involves immune suppression with steroids, which can have limited efficacy and can lead to secondary opportunistic infections. ${ }^{7}$

Riichiro Abe, assistant professor in the Department of Dermatology at the Hokkaido University School of Medicine and a specialist in cutaneous drug eruptions, told SciBX there are no SJS/TEN treatments that are targeted to avoid skin cell apoptosis, which would be the most direct way to treat the disorder.

In their Nature Medicine paper, Yuan-Tsong Chen and colleagues at Academia Sinica now report that granulysin, a cytolytic protein present in SJS and TEN blisters, could be targeted to develop a cure for these conditions. The team showed that granulysin was cytotoxic to keratinocytes and more highly expressed in the blister fluids than other previously identified cytotoxic proteins. The researchers suggested that granulysin could be neutralized to lower keratinocyte cell death.

Using global gene expression profiling, the group assessed levels of various cytotoxic proteins that could be responsible for the cell death that causes the skin lesions. Analysis of blister cells from five patients with confirmed SJS/TEN showed that granulysin expression was significantly higher than that seen in peripheral mononuclear cells from six controls $(\mathrm{p}=1.02 \times 10-5)$. Moreover, disease severity correlated with increasing granulysin expression.

Granulysin levels also were two to four orders of magnitude higher than levels of granzyme B, soluble FasL or other cytotoxic proteins. Depletion of granulysin from the blister fluid with an anti-granulysin antibody decreased cytotoxicity of the fluid against keratinocytes compared with the effects of antibodies against FasL, granzyme B or perforin.

The team thus concluded that granulysin is expressed specifically and at high concentrations in lesions from adverse drug reactions that cause the conditions and is a direct player in skin cell death.

Maria Dolores Ramírez-González, a researcher in this area at the Medical School at the National Autonomous University of Mexico, said the findings were strong and that her only concern "relates to the shortage of information on whole animal experimentation."

Chi-Feng Chang, VP of R\&D at PharmiGene Inc., agreed. "We do need to see more in the animal models. They still need to show that when an antibody to granulysin is injected into the animal, it can prevent disease severity," she said.

PharmiGene markets a CBZ-SJS/TEN Risk Assessment Kit and an Allopurinol-SJS/TEN

Risk Assessment Kit to detect certain genetic variants that increase a patient's risk of reaction from carbamazepine and allopurinol, respectively.

\section{Development avenues}

Chen told SciBX that his group is developing a neutralizing antibody that targets granulysin and is proceeding with animal testing.

"Use of the antibody in SJS or TEN patients could be beneficial, particularly considering that the initial treatment is focused on supportive measures, as if they were burn patients, and the removal of the offending culprit drug," noted Ramírez-González.

Because SJS and TEN can be mistakenly diagnosed-and thus treated-as an infection, other researchers think the nearest-term commercial application of granulysin is for diagnostics.

"When a patient goes to the hospital, it is often unknown whether the cause of the reaction is immune-mediated, such as that caused by SJS/TEN, or the result of an infection," noted Yu-Ligh Liou, director of R\&D at PharmiGene. "The problem is that each of these conditions is treated differently."

For example, said Liou, "one doctor told us that when a patient develops a skin reaction, they need to observe it for a few days to 


\section{TARGETS \& MECHANISMS}

determine the type. If they have granulysin for detection, it is easier to determine whether it is SJS/TEN and requires immune suppression."

Chang added that identification of SJS and TEN at early stages is essential for standard care to have a chance at being effective.

"A patient with SJS needs immune suppression at an early stage to offer the best prognosis, but if the suppression is used unnecessarily or for too long, it could make the patient susceptible to opportunistic infections," she said.

Harald Fricke, COO and CMO of Apogenix $\mathbf{G m b H}$, told SciBX that a better idea for a test for TEN would be to identify the risk of hypersensitivity to drugs before they are prescribed.

"TEN is an extremely rare hypersensitivity reaction of an organism exposed to a drug and occurs after re-exposure," he said. "Such reactions can be prevented by carefully testing for suspected hypersensitivity and simply avoiding re-exposure."

He said that checking patient medical history could prevent reexposure and that he was unaware of any actual tests or markers to tell whether a patient is at risk for SJS or TEN.

Fricke said the company is in discussions to potentially support an investigator-sponsored trial of an undisclosed Apogenix molecule to treat TEN.

Apogenix's lead compound is APG101, a soluble fusion protein combining the extracellular domain of the CD95 receptor and the Fc portion of IgG. The therapeutic is in Phase I testing to treat acute graft-versus-host disease (GvHD).
"The best way to go after an adverse drug reaction is to prevent it from happening at all. But, if that isn't possible, we need to determine how to treat it when it does occur," said Chang.

Chen said a patent application has been filed covering the findings, and the IP is available for licensing.

Martz, L. SciBX 1(45); doi:10.1038/scibx.2008.1093

Published online Dec. 18, 2008

\section{REFERENCES}

1. Chung, W. et al. Nat. Med.; published online Nov. 23, 2008; doi:10.1038/nm.1884

Contact: Yuan-Tsong Chen, Academia Sinica, Nankang, Taipei, Taiwan e-mail: chen0010@ibms.sinica.edu.tw

2. Gomes, E. \& Demoly, P. Curr. Opin. Allergy Clin. Immunol. 5, 309-316 (2005)

3. Roujeau, J. J. Invest. Dermatol. 102, 28S-30S (1994)

4. Sharma, V. et al. Indian J. Dermatol. Venereol. Leprol. 74, 238-240 (2008)

5. Usdin, S. BioCentury 14(14), A1-A6; March 27, 2006

6. Nassif, A. et al. J. Allergy Clin. Immunol. 114, 1209-1215 (2004)

7. Musaka, Y. \& Craven, N. Postgrad. Med. J. 84, 60-65 (2008)

\section{COMPANIES AND INSTITUTIONS MENTIONED}

Academia Sinica, Taipei, Taiwan

Apogenix GmbH, Heidelberg, Germany

Cephalon Inc. (NASDAQ:CEPH), Frazer, Pa.

Food and Drug Administration, Rockville, Md.

Hokkaido University School of Medicine, Sapporo, Japan

National Autonomous University of Mexico, Mexico City, Mexico

Pfizer Inc. (NYSE:PFE), New York, N.Y.

PharmiGene Inc., Taipei, Taiwan 